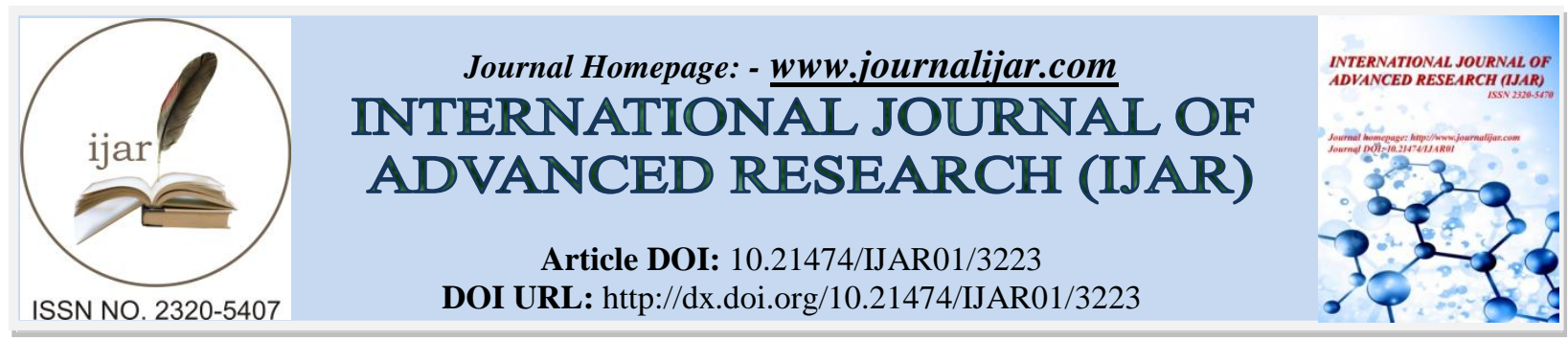

RESEARCH ARTICLE

\title{
3D ULTRASONOGRAPHY DAIGNOSIS OF POLYPOID GALL BLADDER IN DOG.
}

\begin{abstract}
Aissi Adel.
Surgery and Veterinary Imaging Service, Veterinary Department, Veterinary and Agronomic Sciences Institute Batna University, Algeria.
\end{abstract}

\section{Manuscript Info}

Manuscript History

Received: 18 December 2016

Final Accepted: 14 January 2017

Published: February 2017

Key words:-

ultrasound,polyp,gallbladder,dog

\begin{abstract}
Ultrasonographic examination of a five-year-old, male, mixed-breed dog, identified gallbladder wall thickness, accompanied by intraluminal contents suggestive of polyps mass. The liver present normal structure and limits with no mass lesions detected. A cholecystectomy was performed one week after initial case presentation. The gallbladder was enlarged (5 $\mathrm{cm}$ in diameter) with a thickened wall; intraluminal mass 2 $\mathrm{cm}$ in diameter .The fatty mass had a sessile attachment at the neck of the gallbladder and partial occluded the lumen. The surgical removed polyps were later confirmed as cholesterol polyp composed of fatty tisues
\end{abstract}

Copy Right, IJAR, 2017,. All rights reserved.

\section{Introduction:-}

The function of the gallbladder is very closely related to that of the liver. When one is "sick", it is likely to affect the other organ. Drugs that affect one can also affect the other. Therefore it is not uncommon for both diseases to exist in the same dog. The commonest affections of gall bladder and biliary tree included cholangitis, cholecystitis, cholecystolithiasis and choledocholithiasis. Among these the incidence of cholecystolithiasis is very less as evidenced from the published veterinary literatures. The incidence of disorders restricted to the gallbladder and the biliary tree is low, when compared with the parenchymal hepatic affections that occurs in dogs (Veronica et al., 2006). The extrahepatic biliary tract obstruction in dogs is caused most frequently by biliary carcinoma, pancreatic carcinoma, pancreatic disease and intestinal neoplasia. The biliary or intestinal inflammations are less commonly recognized in clinical practice. In human pathology, gall bladder polyps are outgrowths of the gallbladder mucosal wall. They are usually found incidentally on ultrasonography or after cholecystectomy. When detected on ultrasonography, their clinical significance relates largely to their malignant potential. The majority of these lesions are not neoplastic but are hyperplastic or represent lipid deposits(cholesterolosis). On the other hand, imaging In human pathology, gall bladder polyps are outgrowths of the gallbladder mucosal wall. They are usually found incidentally on ultrasonography or after cholecystectomy. When detected on ultrasonography, their clinical significance relates largely to their malignant potential. The majority of these lesions are not neoplastic but are hyperplastic or represent lipid deposits(cholesterolosis). On the other hand, imaging studies alone are insufficiently specific to exclude the possibility of gallbladder carcinoma or premalignant adenomas. Furthermore, even benign lesions can occasionally lead to symptomssimilar to those caused by gallbladder stones. The use of ultrasonography (US) imaging technique became widespread and popular, detection of polypoid lesions of the gallbladder (PLG) has increased significantly (Collett, 1998; Heyder, 1990). Initial studies estimated that PLGs are found in $2.6 \%$ to $12.1 \%$ of cholecystectomy specimens Heyder, 1990). In a large study of 3,608 Danish

\section{Corresponding Author:- Aissi Adel.}

Address:- Surgery and Veterinary Imaging Service, Veterinary Department, Veterinary and Agronomic Sciences Institute Batna University, Algeria. 
subjects, the prevalence of PLG was found to be $4.6 \%$ for men and $4.3 \%$ for women and similar to other studies, these lesions had a higher prevalence in the third and fifth decades of life. Currently, most agree that the average prevalence of PLG in cholecystectomy specimens is about3\% to $12 \%$.the polypoind lesion of the gall bladder rarely observed in veterinary medicine

\section{Description:-}

A five-year-old, male, mixed-breed dog was presented with persistence vomition, anorexia, weakness, for one week. Physical examination revealed icterus mucous membranes and a slightly tensed abdomen with normal hematological profiles. Serum biochemistry abnormalities included increased liver enzyme activities, including alkaline phosphatase $(5,360 \mathrm{U} / \mathrm{L}$; reference range $10-140 \mathrm{U} / \mathrm{L}$ ) as well as hyperbilirubinemia (Total bilirubin $5.6 \mathrm{mg} / \mathrm{dL}$; reference range $0-0.6 \mathrm{mg} / \mathrm{dL}$ ) consistent with hepatocellular damage and cholestasis. with normal levels of blood urea nitrogen $(10 \mathrm{mg} \%)$ and creatinine $(0.2 \mathrm{mg} \%)$ were identified on the serum biochemical analyses. the radiographs demonstrated normal hepatic size and without hepatobilary calcification (Fig.1).Subsequent ultrasonographic examination identified gallbladder wall thickness (Fig.2), accompanied by intraluminal contents suggestive of polyps mass. The liver present normal structure and limits with no mass lesions detected (Fig. 3).

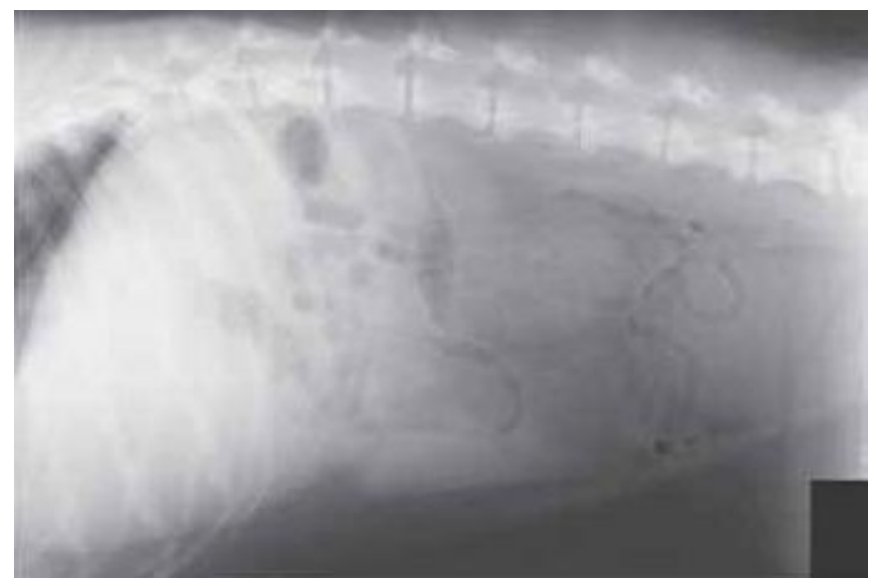

Fig. 1:- Radiograph of hepatic and abdomen cavity demonstrating normancy

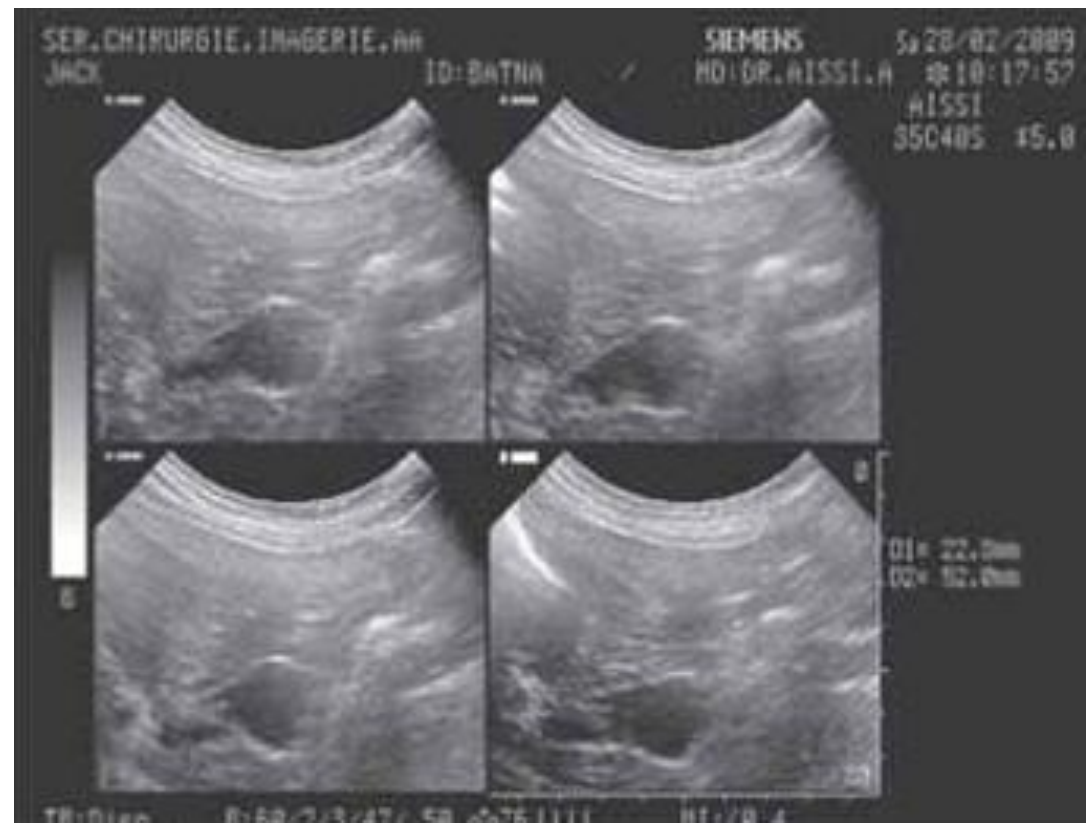

Fig. 2:- Large gall bladder with thickened walls indicating Polyps mass 


\section{D Ultrasonography:-}

After exact tracing of gall bladder and mass structures B-real ultrasonography (2D), 3DU image acquisition was performed as a volume of data with nearly immediate reconstruction and simultaneous display of sectional anatomy in three orthogonal planes (sagittal plane, transverse or coronal plane). Finally a 3D rotating animation of the gall bladder structures at the desired angle could be reconstructed for better visualization and recognition of different parts of mass with gall bladder (Fig. 4).

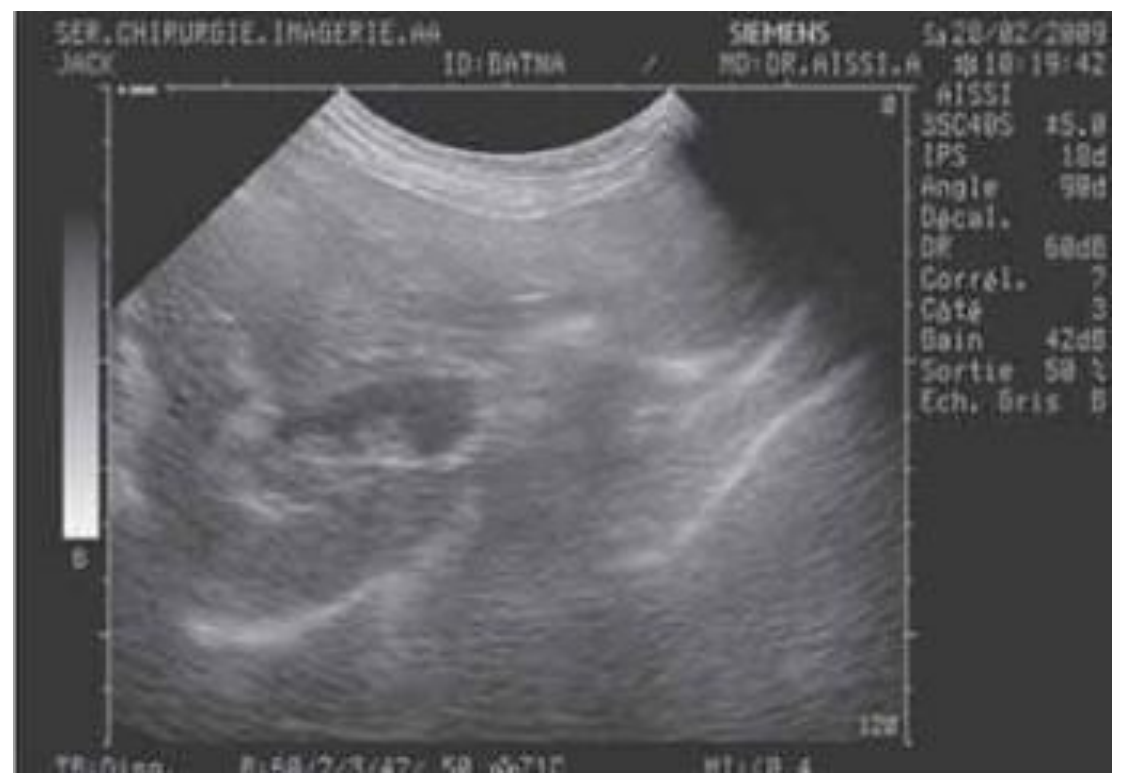

Fig. 3:- Transhepatic ultrasonograms of gall bladder with large mass (Polyp), without a distal acoustic

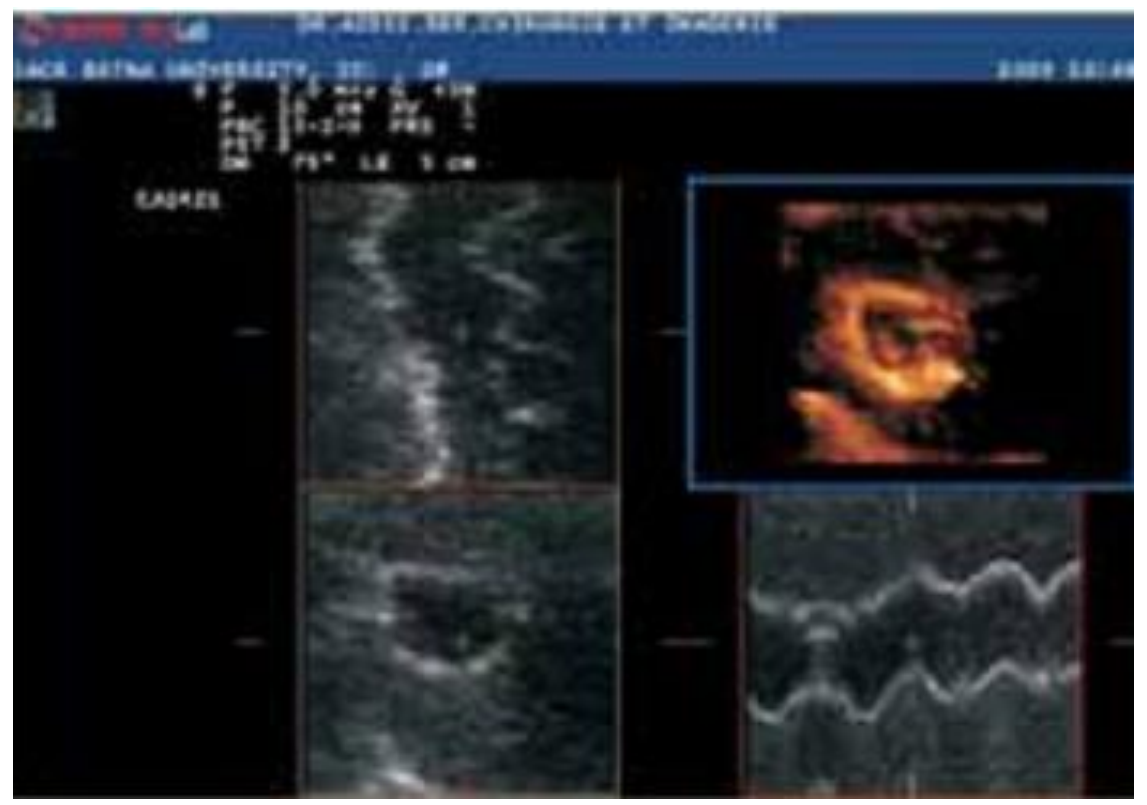

Fig. 4:- Three dimension (3D) reconstitution of gall bladder and intraluminal mass (polyp).

\section{Treatment:-}

On the basis of these findings, a cholecystectomy was performed one week after initial case presentation. The gallbladder was enlarged $(5 \mathrm{~cm}$ in diameter) with a thickened wall and intraluminal mass $2 \mathrm{~cm}$ in diameter. The fatty mass had a sessile attachment at the neck of the gallbladder and partial occluded the lumen. An exploratory 
laparotomy performed at the time of cholecystectomy surgery did not identify other abdominal lesions. Microscopically the mass was composed of fatty tissue (cholesterol polyp).

\section{Discussion:-}

The cause of most gall bladder disease is not well defined in the dog. Many dogs with gall bladder disease have some sort of underlying metabolic or hormonal problem (Weedon; 1984). Both hypothyroidism (low thyroid hormone levels) and hyperadrenocorticism (excessive steroid hormone levels) are associated with an increased risk of developing a biliary disease (Terzi, 2000). These are very rare tumors that account for less than even $1.5 \%$ of all canine tumors. However, secondary hepatobiliary tumors are more common and occur 2.5 times more frequently than primary ones (Collett,1998). In human medicine gallbladder polyps have been observed in 0.004 to 13.8 percent of resected gallbladders (Christensen, 1970; Stringer, 2003), and in 1.5 to 4.5 percent of gallbladders assessed by ultrasonography (Jorgensen, 1990; Veronica, 2006). In one report, no association was observed between the presence of polyps and the patient's age, sex, weight, number of pregnancies, use of exogenous female hormones, or any other risk factors that are generally believed to be associated with gallstones (Stringer, 2003). Gall bladder polypoid lesion has very rarely been reported in dog and domestic animal.

\section{Acknowledgments:-}

The Author is highly thankful to Mr. M. Boukejouta and Hospimed Afric staff for materials and Ultrasonography machine support.

\section{References:-}

1. Christensen AH, Ishak KG. (1970) . Benign tumors and pseudotumors of the gallbladder. Report of 180 cases. Arch Pathol. 90: 423.

2. Collett JA, Allan RB, Chisholm RJ, Wilson IR, Burt MJ, Chapman BA. (1998) . Gallbladder polyps: prospective study. J Ultrasound Med 17: 207-11.

3. Heyder N, Günter E, Giedl J et al. (1990) . [Polypoid lesions of the gallbladder]. Dtsch Med Wochenschr115 $: 243$.

4. Veronica, M., M. Modenato, S. Citi, G. Guidi, (2006) . Choledocholithiasis in a Dog. Annali Fac. Med. Vet. Lix209-18.

5. Jorgensen T, Jensen KH. (1990). Polyps in the gallbladder. A prevalence study. Scand J Gastroenterol25:281.

6. Terzi C, Sökmen S, Seçkin S, Albayrak L, Uðurlu M. (2000) . Polypoid lesions of the gallbladder: report of100 cases with special reference to operative indications. Surgery $127: 622-27$

7. Stringer MD, Ceylan H, Ward K, Wyatt JI. (2003). Gallbladder polyps in children-classification and management. J Pediatr Surg 38:1680.

8. Yang HL, Sun YG, Wang Z. (1992). Polypoid lesions of the gallbladder: diagnosis and indications for surgery. Br J Surg 79:227.

9. Weedon, D. (1984) . Benign mucosal polyps. In pathology of the gallbladder, Mason, New York p.195. 